\title{
On Optimal Ordering of Service Parameters of a Coxian Queueing Model with Three Phases
}

\author{
Vedat Sağlam', Murat Sağır1, Erdinç Yücesoy', Müjgan Zobu² \\ ${ }^{1}$ Department of Statistics, Ondokuz Mayıs University, Samsun, Turkey \\ ${ }^{2}$ Department of Statistics, Amasya University, Amasya, Turkey \\ Email: vsaglam@omu.edu.tr, istatistikci murat@hotmail.com, erdincyucesoy@gmail.com, \\ mujganzobu@hotmail.com
}

Received 8 June 2015; accepted 23 August 2015; published 26 August 2015

Copyright (C) 2015 by authors and Scientific Research Publishing Inc.

This work is licensed under the Creative Commons Attribution International License (CC BY). http://creativecommons.org/licenses/by/4.0/

\section{(c) (i) Open Access}

\section{Abstract}

We analyze a Coxian stochastic queueing model with three phases. The Kolmogorov equations of this model are constructed, and limit probabilities and the stationary probabilities of customer numbers in the system are found. The performance measures of this model are obtained and in addition the optimal order of service parameters is given with a theorem by obtaining the loss probabilities of customers in the system. That is, putting the greatest service parameter at first phase and the second greatest service parameter at second phase and the smallest service parameter at third phase makes the loss probability and means waiting time minimum. We also give the loss probability in terms of mean waiting time in the system. $\alpha_{j}$ is the transition probability from $j$-th phase to $(j+1)$-th phase $(j=1,2)$. In this manner while $\alpha_{1}=0$ and $\alpha_{2} \geq 0$ this system turns into $M|M| 1 \mid 0$ queueing model and while $\alpha_{1} \geq 0, \alpha_{2}=0$ the system turns into $\operatorname{Cox}(2)$ queueing model. In addition, loss probabilities are graphically given in a 3D graph for corresponding system parameters and phase transient probabilities. Finally it is shown with a numeric example that this theorem holds.

\section{Keywords}

Stochastic Coxian Queueing Model, Loss Probability, Limiting Distribution, Optimization, Kolmogorov Equations

\section{Introduction}

Phase-type queueing models are one of the essential parts of the stochastic queueing models. There is an urgent

How to cite this paper: Sağlam, V., Sağır, M., Yücesoy, E. and Zobu, M. (2015) On Optimal Ordering of Service Parameters of a Coxian Queueing Model with Three Phases. Open Journal of Optimization, 4, 61-68.

http://dx.doi.org/10.4236/ojop.2015.43008 
need to construct phase-type distributions for complex representations of queueing models. The recent works being done in this field are: D. R. Cox shows how any distribution having a rational Laplace transform can be represented by a sequence of exponential phases [1]. S. Asmussen, O. Nerman and M. Olsson gave a paper on fitting phase-type distributions with the EM Algorithm [2]. Q. -M. He and H. Zhang presented an algorithm for computing minimal ordered Coxian representations of phase-type distributions whose Laplace-Stieltjes transform had only real poles [3]. The optimal ordering of the tandem server with two stages is given by [4]. R. Marie studied on calculating equilibrium probabilities for Coxian queueing systems in [5]. X. A. Papaconstantinou analyzed the stationary $E_{k} / C_{2} / s$ queueing system in [6]. P. M. Snyder and W. J. Stewart considered two approaches to the numerical solution of single node queueing models with phase-type [7]. In [8], an exact analysis of a fork/join station in a closed queueing network with inputs from servers with two-phase Coxian service distributions is represented. Q. -M. He and H. Zhang studied the approximation of matrix-exponential distributions by Coxian distributions in [9]. M. Fackrell gave a survey of where the phase-type distributions were used in the healthcare industry and purposed some ideas on how they were further utilized [10]. A. B. Zadeh studied a batch arrival queue system with Coxian-2 server vacations and admissibility restricted in [11]. V. Sağlam et al. give a paper on optimization of a Coxian queueing model with two phases in [12].

There is not enough work on the studies of optimizing the orders of service parameters for Coxian queueing model so far. Considering this fact in this paper we analyze a Coxian stochastic queueing model with three phases, and the Kolmogorov equations of this model are constructed, limit probabilities and the stationary probabilities of customer numbers in the system are found. The performance measures of this model are obtained and in addition the optimal order of service parameters is given by a theorem by obtaining the loss probabilities of customers in the system. We also give the loss probability in terms of mean waiting time in the system. Finally it is shown with a numeric example that this theorem holds.

\section{Stochastic Model}

We have obtained stochastic equation systems of a Coxian queueing model with three servers in which the stream is Poisson with $\lambda$ parameter. The service time of any customer at server $i \quad(i=1,2,3)$ is exponential with parameter $\mu_{i}$. Two or more customers can not have service in the system at the same time. Let $\xi_{t}$ be the state of server $1, \eta_{t}$ be the state of server 2 and $\zeta_{t}$ be the state of server 3 at any $t$ time. $\alpha_{j}$ is the transition probability from $j$-th phase to $(j+1)$-th phase $(j=1,2)$ and $1-\alpha_{i}$ be the loss probability of the system. This stochastic queueing model is illustrated in Figure 1. Limit probabilities, differential and difference equations of this system given later.

\section{Limit Probabilities}

Here $\left\{\left(\xi_{t}, \eta_{t}, \zeta_{t}\right), t \geq 0\right\}$ is a three-dimensional Markov chain with continuous parameter and state space is $\Omega=\{(0,0,0),(1,0,0),(0,1,0),(0,0,1)\}$

$$
p_{n_{1}, n_{2}, n_{3}}(t)=\operatorname{Prob}\left\{\xi_{t}=n_{1}, \eta_{t}=n_{2}, \zeta_{t}=n_{3}\right\}, \forall\left(n_{1}, n_{2}, n_{3}\right) \in \Omega
$$

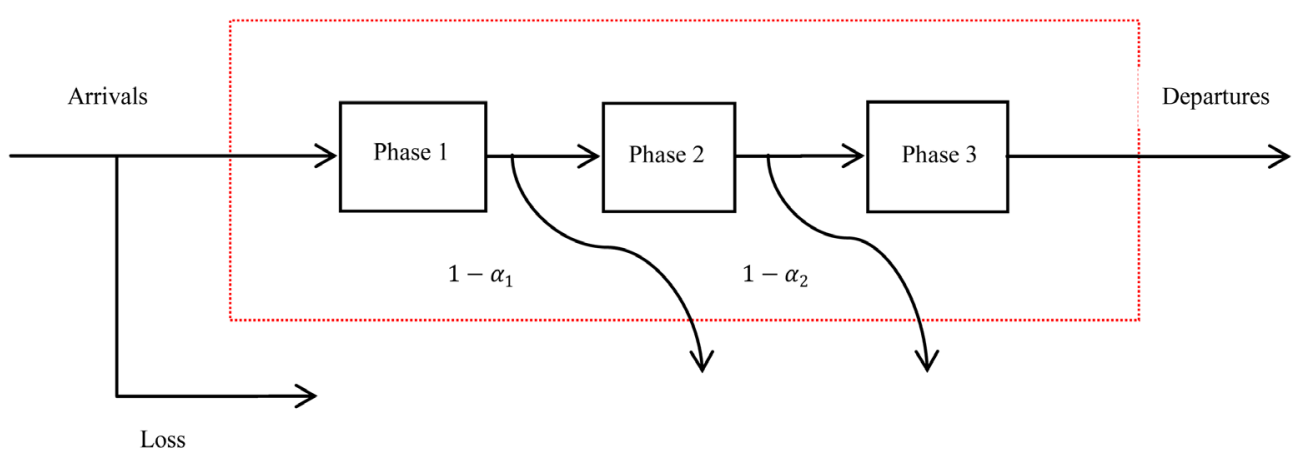

Figure 1. A three phase coxian queueing model. 
Kolmogorov differential equation for these probabilities is obtained. The probabilities of the process $\left\{\left(\xi_{t}, \eta_{t}, \zeta_{t}\right), t \geq 0\right\}$ will be found for $(t, t+h)$, namely

$$
\begin{aligned}
p_{000}(t+h)= & (1-\lambda h+o(h)) p_{000}(t)+\left(1-\alpha_{1}\right)\left(\mu_{1} h+o(h)\right) p_{100}(t) \\
& +\left(1-\alpha_{2}\right)\left(\mu_{2} h+0(h)\right) p_{010}(t)+\left(\mu_{3} h+0(h)\right) p_{001}(t)+o(h) \\
p_{100}(t+h)= & \left(1-\mu_{1} h+o(h)\right) p_{100}(t)+(\lambda h+o(h)) p_{000}(t)+o(h) \\
p_{010}(t+h)= & \left(1-\mu_{2} h+o(h)\right) p_{010}(t)+\alpha_{1}\left(\mu_{1} h+o(h)\right) p_{100}(t)+o(h) \\
p_{001}(t+h)= & \left(1-\mu_{3} h+o(h)\right) p_{001}(t)+\alpha_{2}\left(\mu_{2} h+o(h)\right) p_{010}(t)+o(h)
\end{aligned}
$$

We write Equation (2) as follows as $h \rightarrow 0$

$$
\begin{aligned}
& p_{000}^{\prime}(t)=-\lambda p_{000}(t)+\left(1-\alpha_{1}\right) \mu_{1} p_{100}(t)+\left(1-\alpha_{2}\right) \mu_{2} p_{010}(t)+\mu_{3} p_{001}(t) \\
& p_{100}^{\prime}(t)=-\mu_{1} p_{100}(t)+\lambda p_{000}(t) \\
& p_{010}^{\prime}(t)=-\mu_{2} p_{010}(t)+\alpha_{1} \mu_{1} p_{100}(t) \\
& p_{001}^{\prime}(t)=-\mu_{3} p_{001}(t)+\alpha_{2} \mu_{2} p_{010}(t)
\end{aligned}
$$

Furthermore, it is supposed that limiting distribution of $p_{n_{1}, n_{2}, n_{3}}(t)$ are exist as follow:

$$
\lim _{t \rightarrow \infty} p_{n_{1}, n_{2}, n_{3}}(t)=p_{n_{1}, n_{2}, n_{3}}, \lim _{t \rightarrow \infty} p_{n_{1}, n_{2}, n_{3}}^{\prime}(t)=0 .
$$

Steady-state equations for $\left\{\left(\xi_{t}, \eta_{t}, \zeta_{t}\right), t \geq 0\right\}$ are obtained as following:

$$
\begin{aligned}
& 0=-\lambda p_{000}+\left(1-\alpha_{1}\right) \mu_{1} p_{100}+\left(1-\alpha_{2}\right) \mu_{2} p_{010}+\mu_{3} p_{001} \\
& 0=-\mu_{1} p_{100}+\lambda p_{000} \\
& 0=-\mu_{2} p_{010}+\alpha_{1} \mu_{1} p_{100} \\
& 0=-\mu_{3} p_{001}+\alpha_{2} \mu_{2} P_{010} \\
& \sum_{\left(n_{1}, n_{2}, n_{3}\right) \in \Omega} p_{n_{1}, n_{2}, n_{3}}=1
\end{aligned}
$$

We define $\rho_{i}=\lambda / \mu_{i},(i=1,2,3)$. If we solve Equation (5) under condition (6), we obtain the following three dimension probability function:

$$
p_{n_{1} n_{2} n_{3}}=\left\{\begin{array}{lc}
\frac{1}{1+\rho_{1}+\alpha_{1} \rho_{2}+\alpha_{1} \alpha_{2} \rho_{3}}, & \left(n_{1}, n_{2}, n_{3}\right)=(0,0,0) \\
\frac{\rho_{1}}{1+\rho_{1}+\alpha_{1} \rho_{2}+\alpha_{1} \alpha_{2} \rho_{3}}, & \left(n_{1}, n_{2}, n_{3}\right)=(1,0,0) \\
\frac{\alpha_{1} \rho_{2}}{1+\rho_{1}+\alpha_{1} \rho_{2}+\alpha_{1} \alpha_{2} \rho_{3}}, & \left(n_{1}, n_{2}, n_{3}\right)=(0,1,0) \\
\frac{\alpha_{1} \alpha_{2} \rho_{3}}{1+\rho_{1}+\alpha_{1} \rho_{2}+\alpha_{1} \alpha_{2} \rho_{3}}, & \left(n_{1}, n_{2}, n_{3}\right)=(0,0,1) \\
0, & \text { otherwise }
\end{array}\right.
$$

\section{Obtaining the Measures of Performance}

Let $N$ be the random variable that describes the number of customers in the system. The mean number of costumers:

$$
E[N]=\sum_{\left(n_{1}, n_{2}, n_{3}\right) \in \Omega}\left(n_{1}+n_{2}+n_{3}\right) p_{n_{1} n_{2} n_{3}}=\frac{\rho_{1}+\alpha_{1} \rho_{2}+\alpha_{1} \alpha_{2} \rho_{3}}{1+\rho_{1}+\alpha_{1} \rho_{2}+\alpha_{1} \alpha_{2} \rho_{3}}
$$




$$
\begin{aligned}
& \operatorname{Var}[N]=E\left[N^{2}\right]-E^{2}[N] \\
& =\frac{\rho_{1}+\alpha_{1} \rho_{2}+\alpha_{1} \alpha_{2} \rho_{3}}{1+\rho_{1}+\alpha_{1} \rho_{2}+\alpha_{1} \alpha_{2} \rho_{3}}-\left(\frac{\rho_{1}+\alpha_{1} \rho_{2}+\alpha_{1} \alpha_{2} \rho_{3}}{1+\rho_{1}+\alpha_{1} \rho_{2}+\alpha_{1} \alpha_{2} \rho_{3}}\right)^{2} . \\
& =\frac{\rho_{1}+\alpha_{1} \rho_{2}+\alpha_{1} \alpha_{2} \rho_{3}}{\left(1+\rho_{1}+\alpha_{1} \rho_{2}+\alpha_{1} \alpha_{2} \rho_{3}\right)^{2}}
\end{aligned}
$$

\subsection{The Coxian Queue Using Laplace Transform}

Let $W$ be the random variable that describes waiting time of customers in the system. Laplace transform of $W$

$$
\mathcal{L}_{w}(s)=\frac{\left(1-\alpha_{1}\right) \mu_{1}}{\mu_{1}+s}+\frac{\alpha_{1} \mu_{1}}{\mu_{1}+s} \frac{\left(1-\alpha_{2}\right) \mu_{2}}{\mu_{2}+s}+\frac{\alpha_{1} \mu_{1}}{\mu_{1}+s} \frac{\alpha_{2} \mu_{2}}{\mu_{2}+s} \frac{\mu_{3}}{\mu_{3}+s} .
$$

Mean waiting time in system of a customer for $\operatorname{Cox}(3)$ is found by formula (10)

$$
E[W]=-\left.\frac{\mathrm{d} \mathcal{L}_{W}(s)}{\mathrm{d} s}\right|_{s=0}=\frac{1}{\mu_{1}}+\frac{\alpha_{1}}{\mu_{2}}+\frac{\alpha_{1} \alpha_{2}}{\mu_{3}}
$$

\subsection{The optimization of Measures of Performance}

Loss probability

Let $P_{\text {loss }}$ be the loss probability of customer in the system. In this regards, since there is no queue in the system, loss probability is calculated as following:

$$
P_{\text {loss }}=p_{100}+p_{010}+p_{001} \text {. }
$$

\subsection{Optimal Order of Servers}

We can put three different service parameters to three stages in 3! different position. In this case there are 6 different loss probabilities.

The following theorem is given on minimization of loss probability.

Theorem 1. Putting the greatest service parameter at first phase and the second greatest service parameter at second phase and the smallest service parameter at third phase makes the loss probability minimum. That is,

$$
P_{\text {loss }}^{(1)} \leq P_{\text {loss }}^{(i)}, i=2, \cdots, 6
$$

Proof. Let's suppose $\mu_{1} \geq \mu_{2} \geq \mu_{3}$. In this case we have,

$$
\begin{gathered}
\left(\frac{1}{\mu_{2}}-\frac{1}{\mu_{3}}\right) \leq 0 \\
\alpha_{1}\left(1-\alpha_{2}\right)\left(\frac{1}{\mu_{2}}-\frac{1}{\mu_{3}}\right) \leq 0 \\
\frac{\alpha_{1}}{\mu_{2}}+\frac{\alpha_{1} \alpha_{2}}{\mu_{3}} \leq \frac{\alpha_{1}}{\mu_{3}}+\frac{\alpha_{1} \alpha_{2}}{\mu_{2}} \\
\alpha_{1} \rho_{2}+\alpha_{1} \alpha_{2} \rho_{3} \leq \alpha_{1} \rho_{3}+\alpha_{1} \alpha_{2} \rho_{2} \\
\rho_{1}+\alpha_{1} \rho_{2}+\alpha_{1} \alpha_{2} \rho_{3} \leq \rho_{1}+\alpha_{1} \rho_{3}+\alpha_{1} \alpha_{2} \rho_{2} \\
1+\frac{1}{\rho_{1}+\alpha_{1} \rho_{2}+\alpha_{1} \alpha_{2} \rho_{3}} \geq 1+\frac{1}{\rho_{1}+\alpha_{1} \rho_{3}+\alpha_{1} \alpha_{2} \rho_{2}} \\
\frac{1+\rho_{1}+\alpha_{1} \rho_{2}+\alpha_{1} \alpha_{2} \rho_{3}}{\rho_{1}+\alpha_{1} \rho_{2}+\alpha_{1} \alpha_{2} \rho_{3}} \geq \frac{1+\rho_{1}+\alpha_{1} \rho_{3}+\alpha_{1} \alpha_{2} \rho_{2}}{\rho_{1}+\alpha_{1} \rho_{3}+\alpha_{1} \alpha_{2} \rho_{2}}
\end{gathered}
$$




$$
P_{\text {loss }}^{(1)} \leq P_{\text {loss }}^{(2)} .
$$

Similarly,

$$
\begin{gathered}
\left(1-\alpha_{1}\right)\left(\frac{1}{\mu_{1}}-\frac{1}{\mu_{2}}\right) \leq 0 \\
\frac{1}{\mu_{1}}+\frac{\alpha_{1}}{\mu_{2}} \leq \frac{1}{\mu_{2}}+\frac{\alpha_{1}}{\mu_{1}} \\
\rho_{1}+\alpha_{1} \rho_{2}+\alpha_{1} \alpha_{2} \rho_{3} \leq \rho_{2}+\alpha_{1} \rho_{1}+\alpha_{1} \alpha_{2} \rho_{3} \\
1+\frac{1}{\rho_{1}+\alpha_{1} \rho_{2}+\alpha_{1} \alpha_{2} \rho_{3}} \geq 1+\frac{1}{\rho_{2}+\alpha_{1} \rho_{1}+\alpha_{1} \alpha_{2} \rho_{3}} \\
\frac{1+\rho_{1}+\alpha_{1} \rho_{2}+\alpha_{1} \alpha_{2} \rho_{3}}{\rho_{1}+\alpha_{1} \rho_{2}+\alpha_{1} \alpha_{2} \rho_{3}} \geq \frac{1+\rho_{2}+\alpha_{1} \rho_{1}+\alpha_{1} \alpha_{2} \rho_{3}}{\rho_{2}+\alpha_{1} \rho_{1}+\alpha_{1} \alpha_{2} \rho_{3}} \\
P_{\text {loss }}^{(1)} \leq P_{\text {loss }}^{(3)} .
\end{gathered}
$$

Since,

$$
\alpha_{1}\left(1-\alpha_{2}\right)\left(\frac{1}{\mu_{1}}-\frac{1}{\mu_{3}}\right)+\left(1-\alpha_{1}\right)\left(\frac{1}{\mu_{1}}-\frac{1}{\mu_{2}}\right) \leq 0 \text {, }
$$

we obtain

$$
\begin{aligned}
& \frac{1}{\mu_{1}}+\frac{\alpha_{1}}{\mu_{2}}+\frac{\alpha_{1} \alpha_{2}}{\mu_{3}} \leq \frac{1}{\mu_{2}}+\frac{\alpha_{1}}{\mu_{3}}+\frac{\alpha_{1} \alpha_{2}}{\mu_{1}} \\
& \rho_{1}+\alpha_{1} \rho_{2}+\alpha_{1} \alpha_{2} \rho_{3} \leq \rho_{2}+\alpha_{1} \rho_{3}+\alpha_{1} \alpha_{2} \rho_{1} \\
& \frac{1+\rho_{1}+\alpha_{1} \rho_{2}+\alpha_{1} \alpha_{2} \rho_{3}}{\rho_{1}+\alpha_{1} \rho_{2}+\alpha_{1} \alpha_{2} \rho_{3}} \geq \frac{1+\rho_{2}+\alpha_{1} \rho_{3}+\alpha_{1} \alpha_{2} \rho_{1}}{\rho_{2}+\alpha_{1} \rho_{3}+\alpha_{1} \alpha_{2} \rho_{1}} \\
& \mathrm{P}_{\text {loss }}^{(1)} \leq \mathrm{P}_{\text {loss }}^{(4)} . \\
&\left(1-\alpha_{1}\right)\left(\frac{1}{\mu_{1}}-\frac{1}{\mu_{3}}\right)+\alpha_{1}\left(1-\alpha_{2}\right)\left(\frac{1}{\mu_{2}}-\frac{1}{\mu_{3}}\right) \leq 0 \\
& \frac{1}{\mu_{1}}+\frac{\alpha_{1}}{\mu_{2}}+\frac{\alpha_{1} \alpha_{2}}{\mu_{3}} \leq \frac{1}{\mu_{3}}+\frac{\alpha_{1}}{\mu_{1}}+\frac{\alpha_{1} \alpha_{2}}{\mu_{2}} \\
& \rho_{1}+\alpha_{1} \rho_{2}+\alpha_{1} \alpha_{2} \rho_{3} \leq \rho_{3}+\alpha_{1} \rho_{1}+\alpha_{1} \alpha_{2} \rho_{2} \\
& \frac{1+\rho_{1}+\alpha_{1} \rho_{2}+\alpha_{1} \alpha_{2} \rho_{3}}{\rho_{1}+\alpha_{1} \rho_{2}+\alpha_{1} \alpha_{2} \rho_{3}} \geq \frac{1+\rho_{3}+\alpha_{1} \rho_{1}+\alpha_{1} \alpha_{2} \rho_{2}}{\rho_{3}+\alpha_{1} \rho_{1}+\alpha_{1} \alpha_{2} \rho_{2}} \\
& P_{\text {loss }}^{(1)} \leq P_{\text {loss }}^{(5)} .
\end{aligned}
$$

Finally,

$$
\begin{aligned}
& \left(1-\alpha_{1} \alpha_{2}\right)\left(\frac{1}{\mu_{1}}-\frac{1}{\mu_{3}}\right) \leq 0 \\
& \frac{1}{\mu_{1}}+\frac{\alpha_{1} \alpha_{2}}{\mu_{3}} \leq \frac{1}{\mu_{3}}+\frac{\alpha_{1} \alpha_{2}}{\mu_{1}}
\end{aligned}
$$




$$
\begin{aligned}
\rho_{1}+\alpha_{1} \rho_{2}+\alpha_{1} \alpha_{2} \rho_{3} & \leq \rho_{3}+\alpha_{1} \rho_{2}+\alpha_{1} \alpha_{2} \rho_{1} \\
\frac{1+\rho_{1}+\alpha_{1} \rho_{2}+\alpha_{1} \alpha_{2} \rho_{3}}{\rho_{1}+\alpha_{1} \rho_{2}+\alpha_{1} \alpha_{2} \rho_{3}} & \geq \frac{1+\rho_{3}+\alpha_{1} \rho_{2}+\alpha_{1} \alpha_{2} \rho_{1}}{\rho_{3}+\alpha_{1} \rho_{2}+\alpha_{1} \alpha_{2} \rho_{1}} \\
P_{\text {loss }}^{(1)} & \leq P_{\text {loss }}^{(6)}
\end{aligned}
$$

Corollary. Since,

$$
P_{\text {loss }}=\frac{1}{1+\frac{1}{\lambda E[W]}}
$$

the minimum value which makes $P_{\text {loss }}$ minimum also makes $E[W]$ mininmum.

\section{Numerical Example}

In this section the loss probabilities are calculated for some values of system probabilities and $\alpha_{1}, \alpha_{2}$ probabilities. The calculated loss probabilities are given in Table 1 . For the values $\lambda=2.2, \mu_{1}=8.6 ; \mu_{2}=6.8$, $\mu_{3}=4.4$ and for various values of $\alpha_{1}$ and $\alpha_{2}$ it is seen in Table 1 that $P_{\text {loss }}^{(1)}$ has its minimum value, this shows that Theorem1 holds.

Under condition given in Theorem1, for the values $\lambda=2.2, \mu_{1}=8.6 ; \mu_{2}=6.8, \mu_{3}=4.4$ and for all values of $\alpha_{1}$ and $\alpha_{2}$ in domain set, the loss probabilities are calculated in Table 2 and graphically given in 3D Figure 2 in two different view angle. $P_{\text {loss }}^{(1)}$ is indicated by green surface in this figure. As it is seen in this graph, $P_{\text {loss }}^{(1)}$ is minimum for all values of $\alpha_{1}$ and $\alpha_{2}$. For a customer to have service at each stage it must be $\alpha_{1}=\alpha_{2}=1$ or it must be $\alpha_{1}=0$ for the customer to leave the system after first stage.

\section{Conclusion}

By constructing this stochastic queueing model, transient probabilities are obtained. Depending on these probabilities, mean number of customer in the system, the mean waiting time in this system by Laplace transform and the loss probability of any customer are given. It is shown by Theorem 1 that putting the greatest service parameter at first phase and the second greatest service parameter at second phase and the smallest service parameter at third phase makes the loss probability minimum. For the values $\lambda=2.2, \mu_{1}=8.6 ; \mu_{2}=6.8, \mu_{3}=4.4$ and
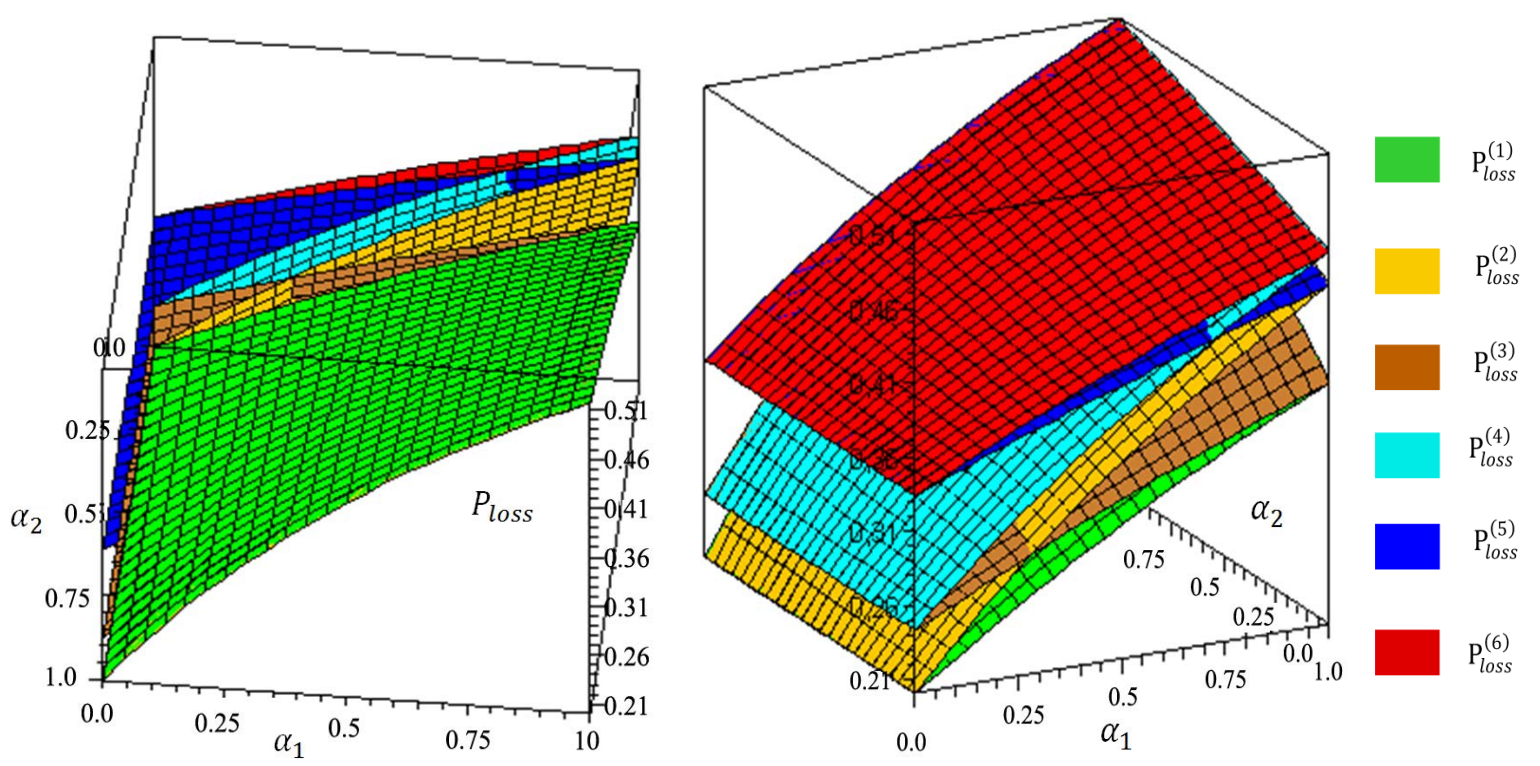

Figure 2. The loss probabilities for $\alpha_{1}$ and $\alpha_{2}$ and system parameters. 
Table 1. Placing the service parameters to phases and corresponding loss probabilities.

\begin{tabular}{cccc}
\hline \multicolumn{3}{c}{ Placing service parameters to phases } & Loss probabilities \\
\hline Phase 1 & Phase 2 & Phase 3 & $P_{\text {loss }}^{(1)}=\frac{\rho_{1}+\alpha_{1} \rho_{2}+\alpha_{1} \alpha_{2} \rho_{3}}{1+\rho_{1}+\alpha_{1} \rho_{2}+\alpha_{1} \alpha_{2} \rho_{3}}$ \\
$\mu_{1}$ & $\mu_{2}$ & $\mu_{3}$ & $P_{\text {loss }}^{(2)}=\frac{\rho_{1}+\alpha_{1} \rho_{3}+\alpha_{1} \alpha_{2} \rho_{2}}{1+\rho_{1}+\alpha_{1} \rho_{3}+\alpha_{1} \alpha_{2} \rho_{2}}$ \\
$\mu_{1}$ & $\mu_{3}$ & $\mu_{2}$ & $P_{\text {loss }}^{(3)}=\frac{\rho_{2}+\alpha_{1} \rho_{1}+\alpha_{1} \alpha_{2} \rho_{3}}{1+\rho_{2}+\alpha_{1} \rho_{1}+\alpha_{1} \alpha_{2} \rho_{3}}$ \\
$\mu_{2}$ & $\mu_{1}$ & $\mu_{3}$ & $P_{\text {loss }}^{(4)}=\frac{\rho_{2}+\alpha_{1} \rho_{3}+\alpha_{1} \alpha_{2} \rho_{1}}{1+\rho_{2}+\alpha_{1} \rho_{3}+\alpha_{1} \alpha_{2} \rho_{1}}$ \\
$\mu_{2}$ & $\mu_{3}$ & $\mu_{1}$ & $P_{\text {loss }}^{(5)}=\frac{\rho_{3}+\alpha_{1} \rho_{1}+\alpha_{1} \alpha_{2} \rho_{2}}{1+\rho_{3}+\alpha_{1} \rho_{1}+\alpha_{1} \alpha_{2} \rho_{2}}$ \\
$\mu_{3}$ & $\mu_{1}$ & $\mu_{1}$ & $P_{\text {loss }}^{(6)}=\frac{\rho_{3}+\alpha_{1} \rho_{2}+\alpha_{1} \alpha_{2} \rho_{1}}{1+\rho_{3}+\alpha_{1} \rho_{2}+\alpha_{1} \alpha_{2} \rho_{1}}$ \\
\hline
\end{tabular}

Table 2. For $\lambda=2.2, \quad \mu_{1}=8.6 ; \mu_{2}=6.8, \mu_{3}=4.4$.

\begin{tabular}{cccccccc}
\hline$\alpha_{1}$ & $\alpha_{2}$ & $P_{\text {loss }}^{(1)}$ & $P_{\text {loss }}^{(2)}$ & $P_{\text {loss }}^{(3)}$ & $P_{\text {loss }}^{(4)}$ & $P_{\text {loss }}^{(5)}$ & $P_{\text {loss }}^{(6)}$ \\
\hline 0 & 0 & 0.203703 & 0.203704 & 0.244444 & 0.24444444 & 0.33333333 & 0.33333333 \\
0.2 & 0 & 0.242722 & 0.262436 & 0.272564 & 0.29752066 & 0.35532233 & 0.36090225 \\
0.4 & 0.8 & 0.352845 & 0.358704 & 0.369425 & 0.37709835 & 0.4137837 & 0.41563943 \\
0.5 & 0.5 & 0.351734 & 0.36976 & 0.365658 & 0.3893066 & 0.41479035 & 0.42053111 \\
0.8 & 0.4 & 0.402855 & 0.431606 & 0.407646 & 0.44610302 & 0.44695789 & 0.45672369 \\
1 & 0 & 0.366825 & 0.430464 & 0.366825 & 0.4516129 & 0.43046357 & 0.4516129 \\
1 & 1 & 0.519078 & 0.519079 & 0.519079 & 0.51907894 & 0.51907894 & 0.51907894 \\
\hline
\end{tabular}

for various values of $\alpha_{1}$ and $\alpha_{2}$ it is seen in Table 1 that $P_{\text {loss }}^{(1)}$ has its minimum value, this shows that Theorem 1 holds. In the case $\alpha_{1}=\alpha_{2}=1$, the loss probabilities are all equal to each other. This is seen in both Table 1 and Graph 1. While $\alpha_{1}=0$ and $\alpha_{2} \geq 0$ this system turns into $M|M| 1|0|$ queueing model and while $\alpha_{1} \geq 0, \alpha_{2}=0$ the system turns into $\operatorname{Cox}(2)$ queueing model. For further studies, higher moments of meanwaiting time in the system can be obtained and by using these moments some various statistical measures can be calculated such as variance, skewness, kurtosis and coefficient of variation. Also this model can be expanded to $k$-phases.

\section{References}

[1] Cox, D.R. (1955) A Use of Complex Probabilities in the Theory of Stochastic Processes. Mathematical Proceedings of the Cambridge Philosophical Society, 51, 313-319. http://dx.doi.org/10.1017/S0305004100030231

[2] Asmussen, S., Nerman, O. and Olsson, M. (1996) Fitting Phase-Type Distributions via the EM Algorithm. Scandinavian Journal of Statistics, 23, 419-441.

[3] He, Q.M. and Zhang, H. (2007) An Algorithm for Computing Minimal Coxian Representations. INFORMS Journal on Computing, 20, 179-190.

[4] Zobu, M., Sağlam, V., Sağır, M., Yücesoy, E. and Zaman, T. (2013) The Simulation and Minimization of Loss Probability in the Tandem Queueing with Two Heterogeneous Channels. Mathematical Problems in Engineering, 2013, Article ID: 529010. 
[5] Marie, R. (1980) Calculating Equilibrim Probabilities for $\lambda(n) / C_{k} / 1 / N$ Queues. ACM Sigmetrics Performance Evaluation Review, 9, 117-125.

[6] Bertsimas, D.J. and Papaconstantinou, X.A. (1988) Analysis of the Stationary $E_{k} / C_{2} / s$ Queueing System. European Journal of Operational Research, 37, 272-287. http://dx.doi.org/10.1016/0377-2217(88)90336-0

[7] Snyder, P.M. and Stewart, W.J. (1985) Explicit and Iterative Numerical Approaches to Solving Queueing Models. Operations Research, 33, 183-202. http://dx.doi.org/10.1287/opre.33.1.183

[8] Krishnamurthy, A., Suri, R. and Vernon, M. (2004) Analysis of a Fork/Join Synchronization Station with Inputs from Coxian Servers in a Closed Queueing Network. Annals of Operations Research, 125, 69-94. http://dx.doi.org/10.1023/B:ANOR.0000011186.14865.19

[9] He, Q.M. and Zhang, H. (2007) Coxian Approximations of Matrix-Exponential Distributions. Calcolo, 44, $235-264$. http://dx.doi.org/10.1007/s10092-007-0139-7

[10] Fackrell, M. (2009) Modelling Healthcare Systems with Phase-Type Distributions. Health Care Management Science, 12, 11-26. http://dx.doi.org/10.1007/s10729-008-9070-y

[11] Zadeh, A.B. (2012) A Batch Arrival Queue System with Coxian-2 Server Vacations and Admissibility Restricted. American Journal of Industrial and Business Management, 2, Article ID: 18843.

[12] Sağlam, V., Uğurlu, M., Yücesoy, E., Zobu, M. and Sağır, M. (2014) On Optimization of a Coxian Queueing Model with Two Phases. Applied and Computational Mathematics, 3, 43-47. 